(1)

${ }^{1}$ Institute of Inflammation and Repair, Wythenshawe Hospital, University of Manchester, Manchester, UK. ${ }^{21 s t}$ Dept of Tuberculosis and Respiratory Care, 1st Medical School and General University Hospital, Charles University in Prague, Prague, Czech Republic. ${ }^{3}$ Dept of Clinical Science, University of Bergen, Bergen, Norway. ${ }^{4}$ Faculty of Medicine, National Heart \& Lung Institute, Imperial College, London, UK. ${ }^{5}$ Dept of Respiratory Medicine, King's College Hospital, London, UK.

\title{
How to keep good clinical records
}

\section{Doing science}

\begin{abstract}
"Verba volant, scripta manent" (spoken words fly away, written words remain)
\end{abstract}

\section{Caius Titus}

Clinical record keeping is an integral component in good professional practice and the delivery of quality healthcare. Regardless of the form of the records (i.e. electronic or paper), good clinical record keeping should enable continuity of care and should enhance communication between different healthcare professionals. Consequently, clinical records should be updated, where appropriate, by all members of the multidisciplinary team that are involved in a patient's care (physicians, surgeons, nurses, pharmacists, physiotherapists, occupational therapists, psychologists, chaplains, administrators or students). Should the need arise patients themselves should have access to their records to be able to see what has been done and what has been considered. Clinical records are also valuable documents to audit the quality of healthcare services offered and can also be used for investigating serious incidents, patient complaints and compensation cases. In this issue of Breathe we will present the importance of keeping good clinical records, ways of facilitating this and an overview of legal aspects linked with clinical record keeping. There is also a list of suggested reading from several countries that may prove useful [1-13].

\section{Why do I need to keep good records?}

Good clinical notes document the medical history of the patient. By documenting all relevant clinical information you are recording this information for future reference. Remember, if you did not write it down, it did not happen. This is of particular relevance in the case of a contested medical decision but most importantly it ensures continuity. Continuity in clinical notes is of vital importance to patient care as, in the current medical environment, many different healthcare professionals are involved in the treatment of a single patient. Making sure that clinical notes are up to date and completed accurately with sufficient information will ensure that the proper information is provided to all relevant healthcare workers and will aid them in potential future decisions. This, in turn, will benefit the patient through less time lost on repeating tests and by averting inaccurate diagnoses or the prescription of inappropriate treatments. Moreover, there is a benefit to the healthcare organisation in that good clinical records facilitate decision making for a single patient, thus freeing up time that can be spent with patients most in need. Finally, poor clinical records might have a profound impact on a patient's lifelong health. Therefore, never forget
Cite as: Mathioudakis $A$, Rousalova I, Gagnat AA, et al. How to keep good clinical records. Breathe 2016; 12: 371-375. 
the seventh principle of the Caldicott report, an NHS report on patient information, which says "the duty to share information can be as important as the duty to protect patient confidentiality". The advantages and disadvantages of keeping good or poor clinical records, respectively, is summarised in Table 1.

\section{What do I need to record and how?}

This is a typical question asked by all trainees, especially at the beginning of their training, when they do their first on-call or acute takes (i.e. unscheduled urgent medical/surgical admissions). Good clinical record keeping under such circumstances can sometimes be really challenging as, for example, in the case of a patient undergoing acute and unexpected deterioration in the middle of the night, with various specialties involved and with several difficult background discussions taking place at the same time with family members. What is deemed to be adequate and what is too much or too little? Usually, there is great variability in the format of entries into clinical notes among different healthcare professionals and hospitals in different countries. This is additionally influenced by the doctor's/healthcare professional's years of experience, any previous incidents (i.e. lessons that have been learnt) and relevant circumstances of the case (acute deterioration of the patient etc).

On a european level there is no set guidance dictating the format of clinical notes. Funded by the health programme of the European Union, an overview of national laws on electronic health records in the member states was published in July 2014. This stated that, to date, all countries used one or several electronic health record systems. It also showed that the requirement as to the content of these records varied broadly from detailed lists to simple references. To tackle this variability, several professional organisations (medical associations, nursing bodies etc) and healthcare trusts have created explicit guidelines about record keeping (what needs to be recorded and in which way) and this is something worth exploring with your current hospital/professional body. Despite its importance, clinical record keeping is often given a low priority. It is common to find illegible entries, offensive comments and missing information, and there is often inconsistency between the entries by different healthcare professionals. Structured information and basic do's and don'ts when keeping clinical records are presented in Table 2 and Table 3.

Every entry in the medical record should be dated, timed ( $24 \mathrm{~h}$ clock) and legible. Each should be signed by the person making the entry and should be made as soon as possible after the event to be documented (e.g. change in clinical state, ward round, investigation etc) and before the relevant staff member goes off duty. If there is a delay, the time of the event and the delay should be recorded as well as the reasons for the delay. Abbreviations should be avoided because they might be ambiguous. For instance, PID could mean prolapsed intervertebral disc or pelvic inflammatory disease. On the other hand, it is acceptable to use short forms in situations where the short form is in common usage in society and would be more easily recognised by the public than the whole term (e.g. HIV, a.m., p.m. etc). It is also important to avoid unnecessary comments in the patient report. Any offensive, personal or humorous comments could damage your credibility. Remember, patients have a right to access their records and a flippant remark in a patient's notes might be difficult to explain.

Medical record entries should be objective. For example, it is risky to refer to a patient as "an alcoholic" or write that they "abuse drugs" without

Table 1 Advantages of keeping good clinical records and the disadvantages of poor clinical records

Good clinical records Poor clinical records

Aid the sharing of relevant information and multidisciplinary team communication

Aid coordination of care

Aid continuity of care

Aid informed decision making for patient management

Improve availability of data for risk assessment

Improve availability of data for route cause analysis in the investigation of serious incidents

Improve audit capabilities

Provide informative evidence in a court of law

Aid targeting of diagnostics and treatment plans without unnecessary repetition

Improve time management
Misinform healthcare professionals and patients

Increase medico-legal risks

Lead to unnecessary repetition of tests or other investigations

Prolong hospital admission

Jeopardise patient care

Lead to serious incidents 
Table 2 Structured information which needs to be included in clinical records

\section{Clinical notes should include}

Patient demographics

Reasons for the current visit

The scope of examination

Positive exam findings

Pertinent negative exam findings

Key abnormal test findings

Diagnosis or impression

Clear management plan and agreed actions

Treatment details and future treatment recommendations

Medication administered, prescribed or renewed and any drug allergies

Written (or oral) instructions and/or educational information given to the patient

Clear documentation and justification for resuscitation status and ceiling of care (if inpatient)

Documentation of communications with patient and family/friends (level of awareness of the situation and acceptance of the plans)

Recommended return visit date

Table 3 Basic do's and don'ts in clinical record entries

\begin{tabular}{ll}
\hline Do & Do not \\
\hline Use timed entries & Use abbreviations \\
Make objective comments & Make offensive, humorous or personal comments \\
Document any noncompliance & Use ambiguous terms \\
$\begin{array}{l}\text { Document oral communications (phone calls, in person conversations } \\
\text { etc) and actions taken }\end{array}$ & $\begin{array}{c}\text { Delete or alter the contents of clinical notes in a way that } \\
\text { is untrackable }\end{array}$ \\
$\begin{array}{l}\text { Document informed consent } \\
\text { State objections regarding care or case management }\end{array}$ \\
\hline
\end{tabular}

objective substantiation of these potentially harmful assertions. Instead, the doctor can conclude that the patient has demonstrated "drug-seeking behaviour". Furthermore, the physician should document objectively what the patient did or said that led the doctor to conclude that the patient demonstrated this behaviour. Use of quotation marks might be helpful in situations where a physical exam fails to provide an objective explanation for a patient's complaints (e.g. I am unable to find an objective explanation for the patient's complaint of "pain"). On the other hand, using too many quotation marks in a report might be distracting. Terms such as "moderate", "heavy", or "occasional" are subject to broad interpretation and the use of quotations is probably unnecessary. Referral recommendations should be documented in unambiguous terms. For example, if the patient needs to understand urgency, this should be obvious from the written comments (i.e. "patient urged to see his gastroenterologist for right upper quadrant abdominal pain and jaundice" instead of "to see GI" and "patient says she will call today for an appointment with orthopaedist for hip pain" in place of "hip pain-needs ortho").

Physicians should document noncompliance in the progress record, such as a patient's failure to follow advice, take medication, obtain requested diagnostic studies, keep an appointment with a consultant, or other actions the patient takes or fails to take that could cause or contribute to an injury or delay in resolution of a medical problem. Patient phone calls should be documented effectively with names, dates and content, including actions taken in response to the patient's call. It is also necessary to make notes regarding patient after-hours phone calls or any consultation with colleagues about the patient case. Informed consent discussions should be documented carefully, as well as discussions with family members, using the same structure as noted above.

For correction of any error draw a single line through the entry, document the correct info, add the date and time, and sign off on the correction. It should 
be possible to track any deletions or alterations back to a named individual at a given time and date. To obtain an idea of the level of a patient's awareness and needs, a physician might want to consider the ICE model (Ideas, Concerns, Expectations) during each visit where: 1) Ideas are "What are your ideas of what is going on?" 2) Concerns are "What are you most worried about?" 3) Expectations are "What are you expecting that I can do?"

\section{Are there any legal issues I should be aware of?}

The content and handling of clinical records is strictly regulated by the law in most countries, not only because they are fundamental to high quality patient care but also because they are increasingly used in the courts and represent an important source of confidential personal information. There are three main pillars of relevant legal obligation. 1) Accuracy and legibility of clinical records content. 2) Confidentiality and data protection. 3) Patients' access to their medical records. While we describe general rules here and give some examples from different countries, we strongly recommend that you should look up the relevant legal requirements in your own country and familiarise yourself with them.

\section{Accuracy and legibility of content}

The "Good Medical Practice" guidelines issued by the General Medical Council UK clearly state that clinical records represent the formal record of a clinician's work and must be clear, accurate, legible and written in a scientific manner. They should include: 1) All relevant clinical findings. 2) A record of the decisions made and actions agreed as well as the identity of who made the decisions and agreed the actions. 3) A record of the information given to patients. 4) A record of any drugs prescribed or other investigations or treatments performed. 5) The identity of who is making the record and when. Similar guidance is provided by other countries, such as Australia (Health Practitioner Regulation (NSW) 2016), most of the United States, France and many others. More important for healthcare providers is that the courts tend to consider that if a medical decision, treatment or procedure is not recorded in the clinical notes then it has not been performed. Thus, in a court of law, it does not matter if you have done your best for your patient unless you have accurately documented this fact. However, the legal burden of proof can depend on the specific setting and circumstances in which the notes are being scrutinised.

\section{Confidentiality and data protection}

Clinical records, including patient identifiers and data on the diagnosis, prognosis or treatment of any patient or subject, are considered confidential globally and can only be shared with the prior written consent of the patient or the subject with respect to whom the record is maintained. Exceptions differ slightly from country to country and it is worth checking the relevant national legislation. For example, the law in the United States suggests that clinical records may be shared without consent, to the extent necessary to treat a medical emergency, with other medical practitioners. They may also be shared with qualified research personnel, to federal, state or local public-health authorities in cases of HIV infection and to state-controlled substance monitoring programmes. Alternatively, in the United Kingdom, confidentiality can be breached in the public interest if failure to disclose such information may expose others to a risk of death or serious harm (e.g. reporting of gunshot and knife wounds, acts of terrorism, risks of serious communicable disease transmission and concerns relating to the ability to drive a motor vehicle). German law is less specific, stating that clinicians can disclose confidential data without consent in order to safeguard a higherranking legally protected interest.

An additional component of confidentiality in the era of technology and the internet refers to data management and data safety. A recent European Union report suggests that 15 out of 28 member countries have adopted specific rules on the content of electronic healthcare records and many of them have specific rules regarding data safety and data sharing. Electronic health records are also strictly regulated in the United States and other countries. Data safety and data sharing law can be very strict in some countries, such as in the United Kingdom for example. The National Health Service in the United Kingdom owns certain e-mail domains, such as “@nhs.net”, that are closely monitored in order to preserve the safety of personal data and medical records. It is not permitted to share patients' data via Gmail, Dropbox, iCloud or personal nonencoded flash drives. Similarly, it is strictly forbidden to share pictures with identifiable patients' data on Facebook, Twitter or other social media platforms.

\section{Patients' access to medical records}

Access to his or her own medical records is an indisputable right of every patient. The process is also regulated in many countries. For example, in United Kingdom, the Data Protection Act 1998 regulates the use of information about living individuals in relation to obtaining, processing, using and disclosing information in general. Everyone responsible for using data has to make sure the information is used fairly, lawfully and for limited, specifically stated purposes in a way that is adequate, accurate, relevant and not excessive. In addition, data should not be kept for longer that is absolutely necessary, should be handled according to people's data protection rights, should be kept safe and secure, and should not be transferred 
outside of the European Economic Area without adequate protection. It also sets out the right for living individuals to be informed that information is being held about them and for what purposes this information will be used. More specifically, the Access to Medical Reports Act 1988 protects the right of an individual to have full access to any medical report related to that individual with minimal exemptions. In many other european countries and american states there are absolutely no exemptions and patients have the right to inspect, copy and access their medical records after a simple written request.

\section{Conflict of interest}

None declared.

\section{Suggested reading}

1. Milieu Ltd, Time.lex. Overview of the national laws on electronic health records in the EU member states and their interaction with the provision of cross-border eHealth services. Brussels, Consumers, health and food executive agency (Chafea), 2014. Available from: http://ec.europa.eu/health/ehealth/docs/ laws_report_recommendations_en.pdf Date last accessed: September 20, 2016

2. Good medical practice. Manchester, General medical council, 2013. Available from: www.gmc-uk.org/static/documents/ content/GMP_.pdf Date last updated: April 29, 2014. Also available from: www.gmc-uk.org/guidance/good_medical_ practice.asp Date last accessed: September 20, 2016.

3. The Code. London, Nursing and midwifery council, 2015; p. 9. Available from: www.nmc.org.uk/globalassets/ sitedocuments/nmc-publications/nmc-code.pdf Date last accessed: November 04, 2016.

4. Confidentiality of certain medical records. US code of laws, title 38, part V, chapter 73, subchapter III, § 7332. Available from: www.gpo.gov/fdsys/pkg/USCODE-2011-title38/pdf/ USCODE-2011-title38-partV-chap73-subchaplll-sec7332.pdf

5. Pullen I, Louden J. Improving standards in clinical record keeping. Adv Psychiatr Treat 2006; 12: 280-6.

6. Confidentiality. Manchester, General medical council, 2009. Available from: www.gmc-uk.org/static/documents/content/ Confidentiality_-_English_1015.pdf Also available from: www. gmc-uk.org/guidance/ethical_guidance/confidentiality.asp. Date last accessed: September 20, 2016.

7. (Model) professional code for physicians in Germany, article 10, obligation to keep medical records. Kiel, 114th German medical assembly, 2011. Available from: www. bundesaerztekammer.de/fileadmin/user_upload/downloads/ MBOen2012.pdf Date last accessed: September 20, 2016.
8. Bundesärztekammer, Kassenärztliche bundesvereinigung. Empfehlungen zur ärztlichen schweigepflicht, datenschutz und datenverarbeitung in der arztpraxis. Deutches Ärzteblatt 2014; 111: A963-A972. Available from: www. bundesaerztekammer.de/fileadmin/user_upload/downloads/ Schweigepflicht_2014.pdf. Date last accessed: September 20, 2016.

9. Health and social care information centre (HSCIC), Academy of medical royal colleges. Standards for the clinical structure and content of patient records. London, HSCIC, 2013. Available from: www.rcplondon.ac.uk/projects/outputs/standardsclinical-structure-and-content-patient-records Date last accessed: September 20, 2016.

10. Clinical guidance (CG2)-record keeping guidelines. Watford, NHS professionals Itd, 2016; version 5. Available from: www.nhsprofessionals.nhs.uk/Download/CG2\%20 -\%20Record\%20Keeping\%20Guidelines\%20V5\%202016. pdf. Date last accessed: November 04, 2016

11. Dossier professionnel ou fiche d'observation, tenu par le médecin. Article R.4127-45 du code de la santé publique. Paris, Conseil national de l'ordre des médecins. Available from: https:// www.conseil-national.medecin.fr/article/article-45-fiche-dobservation-269\#article Date last accessed: September 20, 2016.

12. Code de déontologie médicale. Paris, Conseil national de l'ordre des médecins, 2016. Available from: www.conseilnational.medecin.fr/sites/default/files/codedeont.pdf Date last accessed: September 20, 2016.

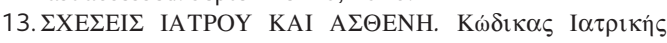

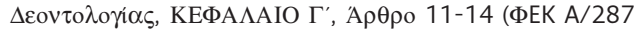

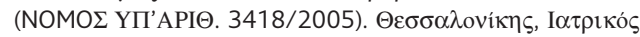

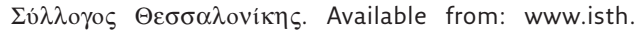
gr/?page=2630. Date last accessed: September 20, 2016. 